\title{
O CONCEITO DE LIMITE E O CONCEITO DE LIMITE SEGUNDO BERTRAND RUSSELL
}

\author{
Douglas Frederico Guimarães Santiago \\ Universidade Federal dos Vales do Jequitinhonha e Mucuri - UFVJM - Brasil \\ Michely Santos Oliveira \\ Universidade Federal dos Vales do Jequitinhonha e Mucuri-UFVJM-Brasil \\ Raquel Anna Sapunaru \\ Universidade Federal dos Vales do Jequitinhonha e Mucuri - UFVJM - Brasil
}

(aceito para publicação em maio de 2016)

\begin{abstract}
Resumo
Este artigo propõe-se explorar o entendimento do conceito de limite, numa abordagem original, a partir de dois pontos de vista distintos: o de Cauchy e o de Russell. Também é feita uma análise do conceito de existência ou não do limite de uma função analítica, com o foco no conceito de limite de Russell, considerandoos conceitos de seção inferior, seção superior, seção inferior extrema, seção superior extrema e oscilação extrema.
\end{abstract}

Palavras-chave: Matemática, História, Limites, Seções, Valores.

\section{[THE CONCEPT OF LIMIT AND THE CONCEPT OF LIMIT ACCORDING BERTRAND RUSSELL]}

\begin{abstract}
This article aims to explore the understanding of the concept of limit, in an original approach from two distinct points of view: Cauchy's and Russell's. Also, it is carried an analysis from the concept of whether or not the limit of a analytical function, with the focus on Russell's concept of limit through the concepts of lower section, upper section, extreme lower section, upper end section and extreme oscillation.
\end{abstract}


Keywords: Mathematics, History, Limits, Sections, Values.

\section{Introdução}

O conceito de limite e sua sistematização lógica alicerçam os cálculos diferencial e integral desde o século XIX. Isso ocorre porque para definir os outros conceitos envolvidos nos cálculos, como os de continuidade, variável, função, quantidade infinitamente pequena e convergência utiliza-se a ideia base do limite. Contudo, o registro evolutivo desse conceito leva a crer o contrário, isto é, que ele, de fato, evoluiu dos cálculos. Historicamente, durante séculos, o conceito de limite foi tratado sem muito rigor, se comparado àquele surgido a partir de Cauchy. Assim, definição verbal de limite de Cauchy surge pela primeira vez em sua obra, Cours d'Analyse de l'École Polytechnique, de 1821, onde o matemático afirma:

"Se os sucessivos valores atribuidos para a mesma variável se aproximam indefinidamente de um valor fixo, tal que finalmente difiram dele tão pouco quanto se queira, esse último valor é chamado limite de todos os outros. Assim, por exemplo, um número irracional é o limite das diversas frações que fornecem os valores mais e mais próximos. $\mathrm{Na}$ geometria, a superfície de um círculo é o limite para o qual convergem as superfícies dos polígonos inscritos, enquanto o número de seus lados cresce mais e mais, etc." (CAUCHY, 1821, p.4)

Nas análises de Grabiner (2005) e Grattan-Guiness (2009), antes que Cauchy entrasse em cena, as ideias que se relacionavam ao conceito de limite eram vagas, subjetivas, pouco rigorosas, intuitivas do ponto de vista geométrico e relativas a um infinito abstrato e obscuro. Porém, para os historiadores Cauchy em sua definição de limite verbal dá um grande passo ao observar que um número irracional é o limite das frações que dele se aproximam. Tudo indica que Cauchy tenha feito uma espécie de formalização da intuição, deixando-a translúcida para seus leitores. ${ }^{1}$

Cabe lembrar que a partir do século XVII, quando os cálculos diferencial e integral foram estabelecidos, Leibniz, Newton, Maclaurin, D'Alambert, L'Huilier, Poinsot, Lagrange e Lacroix expressaram suas próprias ideias de limite, mas essas se mostraram incompletas, por diferentes razões. ${ }^{2}$ Dessa perspectiva, Grattan-Guiness comenta:

"A abordagem de Cauchy [é] baseada numa sólida teoria (e não só uma intuição) de limite [...]; a partir dela ele definiu as noções básicas do cálculo (incluindo a derivada como o valor limitante quociente da

\footnotetext{
${ }^{1} \mathrm{O}$ exemplo da Geometria ser mais adequado àqueles que tomam contato com o conceito de limite pela primeira vez.

${ }^{2}$ Não faz parte do escopo desse artigo explorar essas ideias.
} 
diferença) e das teorias das funções e séries infinitas, para criar 'análise matemática."' (GRATTAN-GUINESS, 2009, p.28)

Definitivamente, o limite moderno inaugurado pelo rigor de Cauchy é um produto do século XIX. ${ }^{3}$ Esse limite abriu inúmeras possibilidades para o desenvolvimento de uma nova Matemática, mais precisa e organizada. ${ }^{4}$ Sob essa luz, a questão da mudança metodológica proposta e empregada por Cauchy, conforme lembrada por Grabiner (2005), torna-se crucial. Entende-se que, caso não houvesse uma mudança metodológica semelhante àquela proposta por Cauchy, o rigor do qual tanto se falou não poderia ter sido empregado, nem no limite, nem nas outras questões matemáticas que o sucederam. Na citação a seguir, Cauchy descreve o rigor de seu método matemático, empregado em sua análise. Nas palavras de Cauchy:

"Quanto aos métodos, eu procurei dar-lhes todo o rigor que exige a geometria, assim como nunca recorri às razões para tirar as generalidade da álgebra. As razões desse tipo, embora sempre suficiente admitidas, especialmente na passagem das séries convergentes às divergentes $e$, das quantidades reais às expressões imaginárias, podem ser consideradas somente, assim me parece, como induções, algumas vezes apropriadas para sugerir a verdade, mas como tendo pouca concordância com a muito elogiada exatidão das ciências matemáticas Deve-se mesmo observar que elas tendem à atribuir as fórmulas algébricas uma extensão indefinida, enquanto que, na realidade, a maioria dessas fórmulas subsidiem somente sob certas condições e, para certos valores de quantidades que eles contém. Determinando essas condições e esses valores e, fixando precisamente o sentido de todas as notações que eu uso, eu faço todas as incertezas desaparecerem; e então as diferentes fórmulas só apresentam as relações entre as quantidades reais, relações que são sempre fáceis de verificar pela substituição de números pelas quantidades." (CAUCHY, 1821, p.ij)

O peso dessa mudança metodológica, ou melhor, do modo de fazer a Matemática, proporcionou um rompimento definitivo entre o século XIX e as ideias e ideais dos séculos passados. De acordo Grabiner (2005), além do inquestionável rigor metodológico apresentado por Cauchy, uma outra diferença entre seu conceito de limite e de seus antecessores residia no seguinte fato, a saber: o uso de desigualdades, como, por exemplo, maior que, menor que, entre outras, em seus teoremas, definições e demonstrações. Vale ressaltar que, no mesmo Cours d'Analyse de l'École Polytechnique, a definição verbal de limite, apresentada anteriormente, foi reescrita em linguagem matemática, utilizando as desigualdades. Na letra de Cauchy:

\footnotetext{
3 “Cauchy (1821), em seu Cours d'Analyse, obra percursora da nova era de rigor que caracterizou o século XIX (...)" (BARONI; OTERO-GARCIA, 2014, p.19)

4 "Cauchy apresentou um novo estilo de rigor que formou o princípio guia para grande parte do desenvolvimento da análise no século XIX. (BARON apud BARONI; OTERO-GARCIA, 2014, p.25)
}

RBHM, Vol. 16, n 31, p. 99-112, 2016 
"Primeiramente, suponha que a quantidade $k$ tenha um valor finito, $e$ seja \& um número tão pequeno quanto se deseja. Uma vez que os valores crescentes de $x$ fazem convergir a diferença, $f(x+1)-f(x)$ para o limite $k$, pode-se dar ao número $h$ um valor suficientemente grande, tal que $x$ sendo igual ou maior que $h$, a diferença em questão está constantemente incluída entre $k-\varepsilon e k+\varepsilon$." (CAUCHY, 1821, p.4849.)

Segundo Katz (2010), Cauchy pesquisou e estabeleceu uma forma de expor correta e rigorosamente os cálculos, objetivando apresentá-los aos seus estudantes de engenharia da École Polytechnique de Paris. Nessa escola, “(...) Cauchy produziu grande parte de seus trabalhos ligados à fundamentação da análise. Isso se deve, em parte, ao compromisso estabelecido pelos professores dessa escola de escreverem textos em todos os níveis (didático, científico)." (BARONI; OTERO-GARCIA, 2014, p.29) Dito isso, no intuito de honrar os esforços de Cauchy, além do conceito de limite já apresentado, oferece-se aqui as definições de variável, ${ }^{5}$ função, ${ }^{6}$ quantidade infinitamente pequena, ${ }^{7}$ continuidade, ${ }^{8}$ e convergência' contidas no livro Cours d'Analyse de l'École Polytechnique, de acordo com

\footnotetext{
5 "Diz-se uma quantidade variável aquela que se considera enquanto passível de receber sucessivamente muitos valores diferentes uns dos outros. Denota-se uma quantidade semelhante por uma letra tomada ordinariamente por meio das últimas letras do alfabeto. Chama-se, ao contrário, quantidade constante, e denota-se uma quantidade semelhante por uma letra tomada ordinariamente por meio das primeiras letras do alfabeto toda quantidade que recebe um valor fixo e determinado." (CAUCHY, 1821, p.4)

6 "Quando quantidades variáveis estão de tal forma ligadas entre si que, o valor de uma delas sendo dada, pode-se concluir que os valores de todas as outras, se concebe essas diversas quantidades expressas por meio de uma entre elas, que recebem então o nome de variáveis independentes; e as quantidades restantes, expressas por meio das variáveis independentes, são o que se chama de funções dessas variáveis. (CAUCHY, 1821, p.19)

7 "Diz-se que uma quantidade variável torna-se infinitamente pequena, quando seu valor numérico decresce indefinidamente de maneira a convergir para o limite zero. É bom observar sobre esse assunto que não se deve confundir um decréscimo constante com um decréscimo indefinido." (CAUCHY, 1821, p.26)

8 "Seja $f(x)$ uma função da variável $x$, e suponhamos que, para cada valor de $x$ intermediários entre dois limites dados, essa função admita constantemente um valor único e finito. Se, partindo de um valor de $x$ compreendido entre esses limites, atribuímos à variável $x$ um acréscimo infinitamente pequeno $\alpha$, a função receberá ela mesma por incremento a diferença $f(x+\alpha)-f(x)$, que dependerá ao mesmo tempo da nova variável $\alpha$ e do valor de $x$. Isso posto, a função $f(x)$ será, entre os dois limites determinados para a variável $x$, função contínua dessa variável, se, para cada valor de $x$ intermediária entre esses limites, o valor numérico da diferença $f(x+\alpha)-$ $f(x)$, decresce indefinidamente com o de $\alpha$. Em outros termos, a função $f(x)$ permanecerá contínua em relação a $x$ entre os limites dados se, entre esses limites, um incremento infinitamente pequeno da variável produzir sempre um incremento infinitamente pequeno da própria função. Dizemos ainda que a função $f(x)$ é, na vizinhança de um valor particular atribuído a variável $x$, função contínua dessa variável, todas as vezes que ela for contínua entre dois limites de $x$, mesmo muito próximos, que incluem o valor a que se referem. Enfim, quando uma função $f(x)$ deixa de ser continua na vizinhança de um valor particular da variável $x$, diz-se que ela se tona então descontínua, e que existe para esse valor particular uma solução de continuidade." (CAUCHY, 1821, p.3435)

9 "Chama-se série uma sucessão indefinida de quantidades $u_{0}, u_{1}, u_{2}, u_{3}, \ldots$ que derivam umas das outras segundo uma lei determinada. Essas mesmas quantidades são os diferentes termos da série que consideramos. Seja $s_{n}=u_{0}$ $+u_{1}+u_{2}+L+u_{n}$ soma dos $n$ primeiros termos, $n$ designando um número inteiro qualquer. Se, para valores de $n$ sempre crescentes, a soma $\mathrm{s}_{\mathrm{n}}$ se aproxima indefinidamente de um certo limite $s$, a série será dita convergente, e o limite em questão se chamará a soma da série. Ao contrário, se, ao mesmo tempo que $n$ cresce indefinidamente, a
} 
a letra de Cauchy. ${ }^{10}$ Mesmo assim, Baroni e Otero-Garcia (2014) observam que nos livros Resumé des leçons sur le calcul infinitésimal, de 1823 e, Leçons sur le calcul differential, de 1829, Cauchy intensificou o tratamento rigoroso e, ao mesmo tempo, refinado de sua análise, ao ensinar aos seus alunos parte de sua obra. ${ }^{11}$ Nesse sentido, parece importante destacar a verdadeira razão de tanto rigor. Para Baroni e Otero-Garcia:

"Como já foi observado, o século XIX é chamado de "era do rigor". Esse rigor podemos compreender como sendo algo que invadiu quase toda a análise, transformando-a na disciplina que hoje em dia é ensinada nas universidades; não foi apenas uma questão de tornar mais claros determinados conceitos básicos e mudar as demonstrações de uns poucos teoremas. Foi um processo de criação que produziu novas áreas $e$ conceitos na matemática como, por exemplo, continuidade uniforme e pontual, convergência uniforme e pontual, compacidade, completude, etc. Mas o rigor em si não era o objetivo dos matemáticos da época; eles estavam voltados a resolver questões técnicas e desenvolver novos teoremas. Um exemplo disso é o interesse despertado pelas séries de Fourier, que acabou mudando velhas ideias a respeito de funções, integral, convergência, continuidade, etc. Também podemos citar o desenvolvimento das equações diferenciais, teoria do potencial e funções elípticas como outras áreas que contribuíram com o processo de rigorização." (BARONI; OTERO-GARCIA, 2014, p.26)

Todavia, apesar da intensa rigorização de seu pensamento matemático, para Cauchy passaram desapercebidos alguns detalhes técnicos, no que diz respeito ao rigor empregado em sua Matemática. ${ }^{12}$ Felizmente, a grande inspiração desse matemático já foi suficiente para estabelecer o contraponto que se deseja entre o seu conceito de limite e o de Russell, fundamentado em seu Principia Mathematica, algumas décadas depois.

Novamente, observa-se que, um dos objetivos dos matemáticos do século XIX era a formalização do então vago conceito de limite. Assim, no final do século XIX, surgia a conquista desse rigor conceitual em relação aos cálculos diferencial e integral. Isso envolvia

soma $\mathrm{S}_{\mathrm{n}}$ não se aproxima de qualquer limite fixo, a série será divergente e não terá soma. Nos dois casos, o termo que corresponde ao índice $n$, a saber $u_{n}$, será aquele que chama-se de termo geral. É suficiente que se dê o termo geral em função do índice $n$, para que a série seja completamente determinada.” (CAUCHY, 1821, p.123)

${ }^{10}$ Optou-se nesse artigo por não apresentar a derivada e a integral de Cauchy, que se encontram detalhadas no livro Resumé des leçons sur le calcul infinitesimal.

${ }^{11}$ Segundo Katz (2010), o total de obras escritas por Cauchy é de 789 artigos, em vinte e sete volumes, reunidas nas CEuvres Complètes d'Augustin Cauchy.

${ }^{12}$ Baroni e Otero-Garcia (2014) ao discorrerem sobre o "movimento do rigor" destacam a participação de Weierstrass. Esse matemático "(...) teve papel fundamental no desenvolvimento da análise, especialmente no uso dos épsilons e deltas, característica do formalismo que a disciplina possui até hoje." (BARONI; OTEROGARCIA, 2014, p.77) Todavia, coube a Abel mostrar que Cauchy provou incorretamente que o limite de uma série convergente de funções contínuas era também contínua. "Mas parece-me que este teorema [de Cauchy] admite exceções. Por exemplo, a série $\sin x-\frac{1}{2} \sin 2 x+\frac{1}{3} \sin 3 x-\cdots$ é descontínua para cada valor de $(2 m+1)$ $\pi$ de $x$, onde $m$ é um inteiro. É bem sabido que há muitas séries com propriedades similares." (ABEL apud KATZ, 2010, p. 909)

RBHM, Vol. 16, nº 31, p. 99-112, 2016 
os conceitos de limite, as definições de variável, função, quantidade infinitamente pequena, continuidade e convergência, entre outros. Em meio aos interessados nesse assunto, havia um grupo especial, cujo enfoque se direcionava mais para a discussão em torno dos fundamentos da aritmética do que para a solução de problemas técnicos. Esse programa foi batizado de "Aritmetização da Análise”. Segundo Katz (2010), nele, matemáticos como Frege, Cantor e, principalmente, Russell, ansiavam por agenciar a própria aritmética em uma base mais profunda, tornando-a mais expressiva e contundente. Esses matemáticos, na segunda metade do século XIX, tomaram para si a missão de reduzir o conceito de número natural ao conceito lógico de classe e, como desejava Cantor, definir os números em termos de conjuntos. Portanto, a lógica das classes revelava-se como a teoria mais apropriada para a investigação dos fundamentos da Matemática. Nesse pano de fundo, destaca-se o conceito de limite de Russell.

O presente artigo pretende debruçar sobre o conceito de limite de forma diferente, com diferentes pontos de vista, a saber: o proveniente do conceito de Cauchy e a forma que Russell usou para introduzi-lo no Capítulo XI do Introdução à Filosofia da Matemática, de 1919, e na Seção C do Volume 2 do Principia Mathematica, finalizado em 1927, apesar de ter sido apresentado pela primeira vez em 1910. (RUSSELL; WHITEHEAD, 2013 (v1), p.4) Outrossim, propõe-se analisar o conceito de existência ou não do limite de uma função conforme a ideia de Russell, considerando os conceitos de seção inferior, seção superior, seção inferior extrema, seção superior extrema e oscilação extrema. Reforça-se aqui que o objetivo não é escolher o melhor conceito, mas entendê-los profundamente, por meio de uma comparação simples, ombro a ombro.

\section{O limite segundo Russell}

Estudar um conceito de forma diferente, com diferentes pontos de vista, sempre auxilia no seu entendimento. O conceito formal de limite à esquerda de uma função normalmente é visto nos livros de cálculo da seguinte forma:

Diz-se que o limite de uma função, $f: \mathbb{R} \rightarrow \mathbb{R}$ quando $x$ tende a c pela esquerda, existe e é igual a $L$ quando, para todo $\varepsilon>0$, existe $\delta>0$ tal que se $x \in(c-\delta, c)$ então $f(x) \in$ $(L-\varepsilon, L+\varepsilon)$.

De forma análoga, pode-se definir o que é o limite à direita, sendo que o limite só existirá se ambos existirem e forem iguais. Assim, como descrito nos objetivos desse artigo, deseja-se analisar o conceito de existência ou não do limite de uma função à moda de Russell. Logo, é preciso entender e utilizar os conceitos de seção inferior, ${ }^{13}$ seção superior, seção inferior extrema, seção superior extrema e oscilação extrema. Para tal, seguem as definições:

Definição 1. A seção inferior de uma função $f$ sobre o intervalo $(a-\varepsilon, a)$, com $\varepsilon>0$, é o conjunto dos números reais que são menores ou iguais que algum $f(x)$ para $x \in(a-$ $\varepsilon, a)$.

Definição 2. A seção inferior extrema é a interseção das seções inferiores para todo $\varepsilon>0$.

${ }^{13}$ Nesse caso, Russell se refere apenas à seção, mas o nome seção inferior se ajusta melhor à ideia. 
Definição 3. A seção superior de uma função $f$ sobre o intervalo $(a-\varepsilon, a)$, com $\varepsilon>0$, éo conjunto dos números reais que são maiores ou iguais que algum $f(x)$ para $x \in(a-$ $\varepsilon, a)$.

Definição 4. A Seção superior extrema é a interseção das seções superiores para todo $\varepsilon>0$.

Definição 5. A Oscilação extrema é o conjunto dos números reais pertencentes tanto à seção inferior extrema quanto a seção superior extrema. (RUSSELL, 1966, p.111; RUSSELL; WHITEHEAD, 2013 (v2), p.727).

Segundo Russell, o limite à esquerda existe quando a seção superior extrema e a seção inferior extrema são não-vazias e a oscilação extrema não contém elemento algum ou apenas $;{ }^{14}$ de forma análoga, ocorre para o limite à direita, considerando o intervalo $(a, a+$ $\varepsilon)$ ao invés do intervalo $(a-\varepsilon, a)$. Na sequência, por meio dos exemplos que serão apresentados, pode-se ter uma ideia de como essas definições funcionam para explicar o conceito de limite, cabendo ao leitor mais interessado fazer a demonstração de que elas realmente funcionam.

\section{A formulação de Russell vs. Conceitos modernos}

Nesse ponto, a forma como Russell introduziu o conceito de limite será comparada com algumas formas diferentes de verificá-lo, baseadas em aspectos da teoria moderna do cálculo em uma variável, que se originaram do conceito de limite de Cauchy. Para isso, serão explorados os exemplos de três funções clássicas do cálculo, uma função afim, na qual o limite em questão existirá, a função $\mathrm{f}(\mathrm{x})=\operatorname{sen}(1 / \mathrm{x})$ que não possui limite para $x=0$, e a função $f(x)=1 / x$, que possui um comportamento assintótico para $x=0$. Vale lembrar que Russellao introduzir seu conceito de limite, não faz referência explícita ao caso de funções com comportamento assintótico.

\footnotetext{
${ }^{14}$ Russell não especifica a restrição da seção superior extrema e da seção inferior extrema serem não vazias, mas isso se faz necessário no caso de funções com pontos singulares, como será mostrado nos exemplos selecionados.
}

RBHM, Vol. 16, n 31, p. 99-112, 2016 


\section{Limite quando $x \rightarrow 1$ da função $f(x)=2 x+1$}

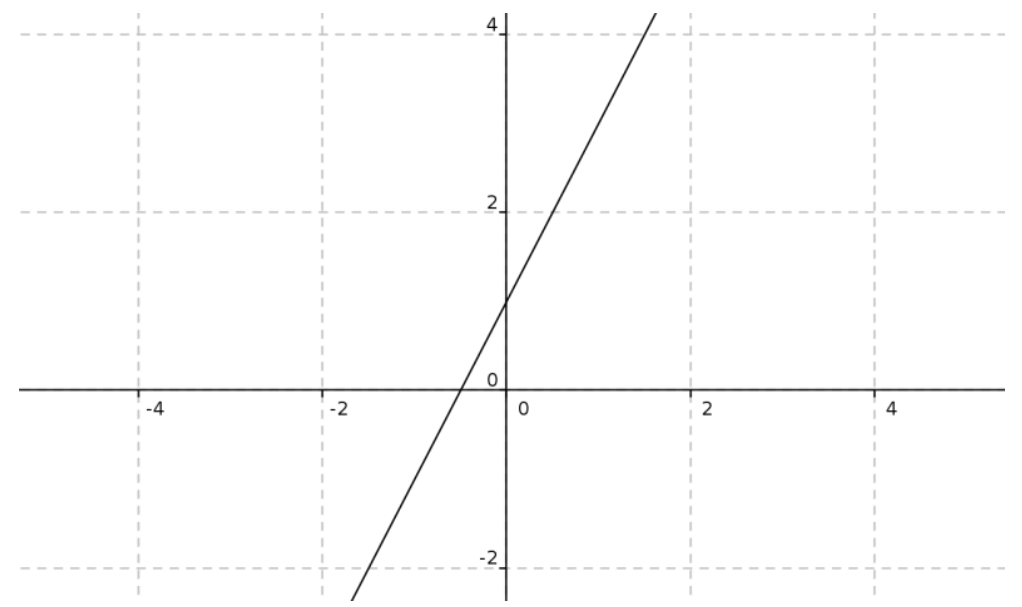

Pode-se usar a definição usual de continuidade para verificar que o limite quando $x \rightarrow 1$ de $f(x)=2 x+1$ é $f(1)=3$, o que faz da função uma função contínua em $x=1$ (GUIDORIZZI, 2001, p.60-70). ${ }^{15}$ Assim, deve-se provar que para todo $\varepsilon>0$ dado, consegue-se um $\delta>0$ tal que:

$1-\delta<x<1+\delta=>f(1)-\varepsilon<f(x)<f(1)+\varepsilon$

Como se quer que:

$3-\varepsilon<2 x+1<3+\varepsilon$

manipula-se, então, essas equações para estabelecer uma condição sobre $x$. Primeiro, subtrai-se tudo por $-1 \mathrm{e}$, assim, tem-se:

$2-\varepsilon<2 x<2+\varepsilon$

Dividindo por 2, tem-se:

$1-\frac{\varepsilon}{2}<x<1+\frac{\varepsilon}{2}$

Assim, para $\delta=\frac{\varepsilon}{2}$, vale:

$1-\delta<x<1+\delta=>f(1)-\varepsilon<f(x)<f(1)+\varepsilon$

Logo, pode-se concluir que:

$\lim _{x \rightarrow 1} f(x)=f(1)=3$

Todavia, segundo Russell, determina-se a existência do limite à esquerda considerando os intervalos $(1-\varepsilon, 1)$. Posto, tem-se:

$f(1-\varepsilon)=2(1-\varepsilon)+1=3-2 \varepsilon$ e $f(1)=3$

Como a função é crescente, tem-se que a seção inferior é $(-\infty, 3)$ e a seção superior é $(3-2 \varepsilon,+\infty)$. A tabela abaixo mostra o que ocorre com as seções inferior e superior para diferentes valores de $\varepsilon$ :

${ }^{15}$ Trata-se da seção onde o autor discorre sobre funções contínuas. 
Tabela 1: Seções inferior e superior para diferentes valores de $\varepsilon$

\begin{tabular}{|c|c|c|}
\hline$\varepsilon$ & Seção inferior & Seção superior \\
\hline 1 & $(-\infty, 3)$ & $(1,+\infty)$ \\
\hline 0,1 & $(-\infty, 3)$ & $(2.8,+\infty)$ \\
\hline 0,01 & $(-\infty, 3)$ & $(2.98,+\infty)$ \\
\hline
\end{tabular}

Observa-se que, ao considerar a interseção desses conjuntos para todos os valores de $\varepsilon$ possíveis, a seção inferior extrema é $(-\infty, 3)$ e a seção superior extrema é $[3,+\infty)$, logo a oscilação extrema é o conjunto vazio. Como a oscilação extrema não possui termo algum, a função possui limite à esquerda, que nesse caso é 3. De forma análoga, procede-se para o limite à direita.

\section{2) Limite quando $x \rightarrow 0$ da função $f(x)=\operatorname{sen}(1 / x)$}

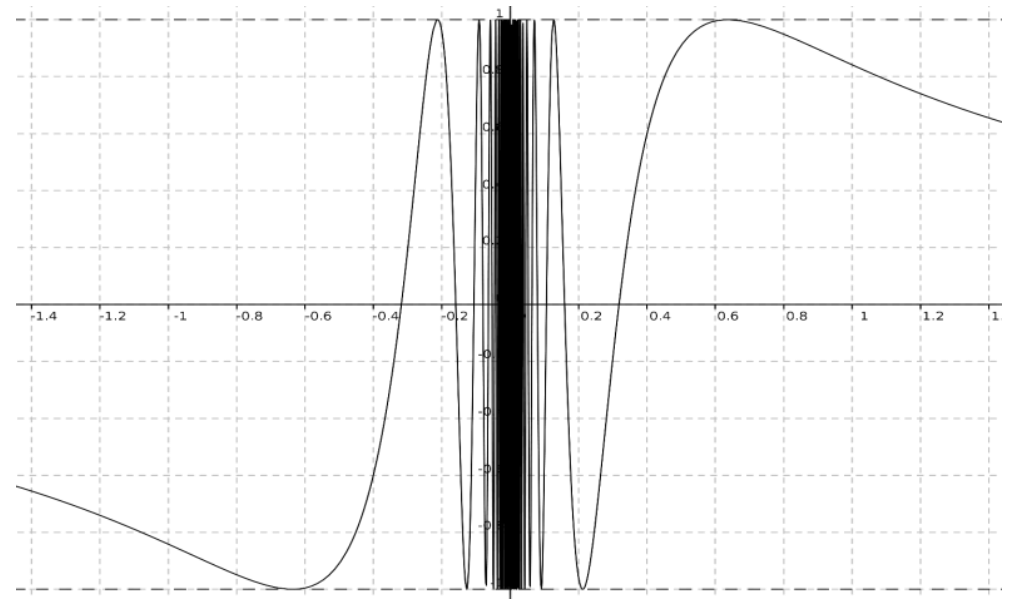

Empregando as ideias usuais de limite, pode-se afirmar que não existe $\lim _{x \rightarrow 0} \operatorname{sen}(x)$. Considere a sequência $\left\{x_{k}\right\}$, na qual $x_{k}=\frac{2}{(2 k+1) \pi}$. Em qualquer intervalo $(0, \varepsilon)$, com $\varepsilon>0$, sempre há pelo menos dois termos da sequência, $x$ e $\bar{x}$, tal que $f(x)=1$ e $f(\bar{x})=-1$. Assim, não há como o limite à direita da função existir. De forma análoga, é feito para o limite à esquerda.

Vamos então verificar, de acordo com Russell, a não existência do limite à esquerda. Para determinar a seção inferior, analisamos valores de $x$ no intervalo fixo $(-\varepsilon, 0)$. Nesse intervalo, o máximo que a função atinge é 1 ; logo a seção inferior é $(-\infty, 1]$. Em seguida, toma-se o menor valor que a função atinge que, no exemplo, vai valer -1 e tudo que está acima dele; logo, a seção superior é $[-1, \infty)$. Observa-se que nesse 
exemplo, tanto a seção superior, quanto a inferior não dependem de $\varepsilon$; $\operatorname{logo}$ as seções superiores e inferiores extremas serão as mesmas e a oscilação extrema será $[-1,1]$.

Tem-se, então:

a) Seção inferior: $(-\infty, 1]$ e seção superior: $[-1, \infty)$.

b) Seção inferior extrema: $(-\infty, 1]$ e seção superior extrema: $[-1, \infty)$

c) Oscilação extrema: $[-1,1]$

Por fim, conclui-se que o limite não existe, pois a oscilação extrema contém mais de um ponto.

3) Limite quando $x \rightarrow 0$ da função $f(x)=1 / x$

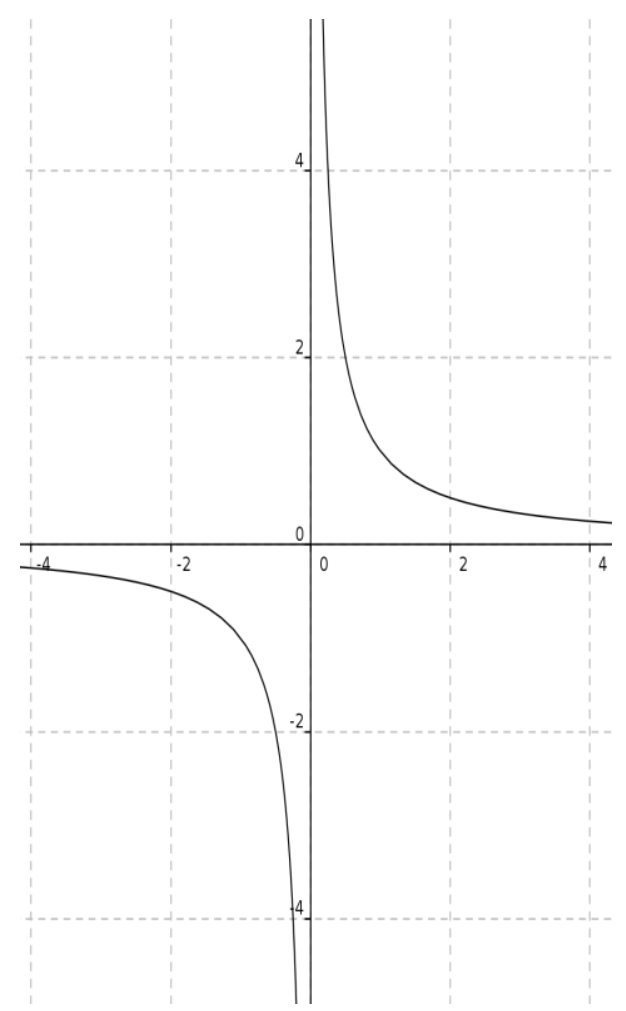

Para verificar, à moda de Cauchy, que o limite à esquerda não existe, fixa-se $\varepsilon=1$. Se um número real $c$ fosse o limite, então, em particular, para este $\varepsilon$ dado, existiria $\gamma>0$, tal que para todo $x \in(-\gamma, 0), f(x) \in(c-1, c+1)$. Ocorre que para $c \geq 1$ isto é claramente impossível, pois em $(-\gamma, 0)$, a função só atinge valores negativos. Para $c<1$, tem-se $c-1<0$ e, consideram-se dois casos: 
1) Se $\gamma>\frac{1}{|c-2|}$, toma-se $x=-\frac{1}{|c-2|}$. Tem-se, então, $x \in(-\gamma, 0)$ e $f(x)=$ $-|c-2|$ que, por sua vez, não pertence ao intervalo $(c-1, c+1)$, pois para $c<1$, tem-se $-|c-2|<-|c-1|$.

2) Se $\gamma \leq \frac{1}{|c-2|}$, tom-se $x=-\frac{\gamma}{2}$. Também, tem-se $f(x)=-\frac{2}{\gamma} \leq-2|c-2|<$ $-2|c-1|$ que, por sua vez, não pertence ao intervalo $(c-1, c+1)$.

Analogamente, verifica-se que não existe o limite à direita.

Já utilizando as ideias de Russell, considera-se para $\varepsilon>0$, o intervalo $(-\varepsilon, 0)$ e tem-se $f(0-\varepsilon)=-1 / \varepsilon$. Pela definição, as seções inferior e superior são, respectivamente, $(-\infty,-1 / \varepsilon)$ e $(-\infty,+\infty)$. Logo, a seção inferior extrema é o conjunto vazio e a seção superior extrema é $(-\infty,+\infty)$. Dessa forma, a interseção da seção superior extrema com a seção inferior extrema, isto é, a oscilação extrema, é vazia. Segundo a discussão inicial de Russell sobre a existência ou não do limite, esse deveria existir nesse caso, mas sabe-se que isso não ocorre. Dessa forma, a ideia primeiramente apresentada por Russell sobre o limite parece considerar apenas funções em que os limites superiores e inferiores extremos sejam não vazios, descartando assim as funções assintóticas. Para que a ideia de Russell continue sendo válida, nesse caso, deve-se acrescentar a condição que ambas, a seção inferior extrema e a seção superior extrema, sejam não vazias, isto é, o limite à esquerda só existe quando a seção superior extrema e a seção inferior extrema são não vazias e, a oscilação extrema não contém elemento algum, ou apenas um elemento. Nesse exemplo, como a seção inferior extrema não possui elementos, a função tem um comportamento assintótico quando x tende a $0 ; \operatorname{logo}$, o limite pela esquerda não existe.

De forma análoga, pode-se analisar o intervalo $(0, \varepsilon)$. Assim, a seção inferior será $(-\infty,+\infty)$ e a seção superior, $(-\infty, 1 / \varepsilon)$; logo a seção inferior extrema será $(-\infty,+\infty)$ e a seção superior extrema será o conjunto vazio. Como a seção superior extrema não possui elementos, então a função possui um comportamento assintótico quando $x$ tende a 0 e o limite à direita também não existe.

\section{Como determinar qual o limite usando a formulação apresentada por Russell}

Tanto a formulação moderna do conceito de limite, quanto a formulação primeiramente apresentada por Russell em seu Principia Mathematica não exploram a importante questão de como determiná-lo. Na formulação moderna, dado um número $L$, candidato a ser o limite, pode-se verificar se realmente ele é o limite ou não. Na formulação primeiramente apresentada por Russell, tem-se que determinar a oscilação extrema, e caso esse conjunto seja vazio, ou contenha apenas um elemento, o limite existe. Determinar se o limite existe ou não é uma importante questão, mas caso ele exista, surge outra questão, tão importante quanto a anterior, que é saber qual é o limite em questão. Para determinar esse limite, aproveitando a formulação de Russell, enuncia-se, então, a seguinte proposição:

Proposição: Seja a função $f: \mathbb{R} \rightarrow \mathbb{R}, A$ e $B$ respectivamente a seção inferior extrema e superior extrema sobre o intervalo $(c-\varepsilon, c)$. Se $A$ e $B$ são ambos não vazios e tem interseção vazia ou contendo apenas um ponto, então o supremo de $A$ e o ínfimo de $B$ 
existem, e o limite da função quando $x \rightarrow c^{-}$é Sup $(A)$. A prova dessa proposição se dá por meio dos seguintes argumentos:

Em primeiro lugar, se ambas as seções inferiores e superiores extremas são não vazias, então a seção inferior extrema é limitada superiormente, e a seção superior extrema é limitada inferiormente, pois caso a seção inferior extrema não fosse limitada superiormente, a seção superior extrema seria vazia e vice-versa. Dessa forma, prova-se que existem o supremo de $A$ e o ínfimo de B. Além disso, também tem-se $\operatorname{Sup}(A)=\operatorname{Inf}(B)$, pois, por definição de seção inferior e seção inferior extrema, existe $\varepsilon_{1}$, tal que $f(x)<$ $\operatorname{Sup}(A)$, para algum $x \in(c-\varepsilon, c)$, para todo $\varepsilon \leq \varepsilon_{1}$, logo por definição de seção superior $\mathrm{e}$ seção superior extrema, $\operatorname{Sup}(A)$. Se $\operatorname{Inf}(B)<\operatorname{Sup}(A)$, então a interseção de $A$ e $B$ conteria mais de um ponto e, com isso, $\operatorname{In} f(B)=\operatorname{Sup}(A)$. Para provar que o limite da função quando $x \rightarrow c^{-}$é $\operatorname{Sup}(A)$, toma-se $\sigma>0$ e o intervalo $(\operatorname{Sup}(A)-\sigma, \operatorname{Sup}(A)+\sigma)$. Como $\operatorname{Sup}(A)=\operatorname{Inf}(B)$, então $\operatorname{Sup}(A)-\sigma$ é uma cota inferior para $B$, então existe $\varepsilon_{1}$, tal que $f(x)>\operatorname{Sup}(A)-\sigma$, para todo $x \in\left(c-\varepsilon_{1}, c\right)$. De forma análoga, como $\operatorname{Sup}(A)+$ $\sigma$ é uma cota superior para $A$, existe $\varepsilon_{2}$, tal que $f(x)<\operatorname{Sup}(A)+\sigma$ para todo $x \in(c-$ $\left.\varepsilon_{2}, c\right)$. Por fim, basta então escolher $\varepsilon=$ mínimo $\left\{\varepsilon_{1}, \varepsilon_{2}\right\}$ e tem-se que para todo $x \in$ $(c-\varepsilon, c), f(x) \in(\operatorname{Sup}(A)-\sigma, \operatorname{Sup}(A)+\sigma)$.

\section{Conclusão}

Resgatando o objetivo geral desse artigo, isto é, lançar um novo olhar sobre o conceito de limite, explorando díspares pontos de vista, entende-se que isso foi mostrado de modo claro e distinto, ou seja, explorou-se, satisfatoriamente, o que era necessário para proporcionar o entendimento da dimensão do conceito de limite, de duas formas emblemáticas. Assim, tanto na visão de Cauchy, quanto na visão de Russell, o conceito de limite se revela como uma ferramenta que auxilia o entendimento dos cálculos diferencial e integral.

Considerando o objetivo principal desse artigo, ou seja, comparar as formas usuais utilizadas para determinar a existência ou não do limite de uma função, cuja base encontrase na ideia de Cauchy, com a forma primeiramente apresentada por Russell em sua obra Principia Mathematica, destacam-se alguns pontos:

1. As formas usuais, baseadas na formulação de Cauchy, são úteis nos casos em que se tem um candidato $L$, a ser o limite da função e, então, precisa-se verificar que esse é, realmente, o caso. Para atingir esse objetivo, a dificuldade consiste em dado qualquer valor positivo, $\sigma$, determinar de acordo com a função dada, um valor positivo $\gamma$, que seja função de $\sigma$, tal que, no caso de limites à esquerda, garanta que $f(x) \in(L-\sigma, L+\sigma)$. A dificuldade dessa tarefa vai depender da função dada.

2. O limite, como foi introduzido primeiramente por Russell em sua obra, por meio dos conceitos de seção superior, inferior, seção superior extrema, inferior extrema e oscilação extrema, em princípio, se aplicaria apenas para determinar a existência ou não do limite. $\mathrm{O}$ limite russeliano tem a vantagem de não precisar de um candidato inicial $L$ para ser o limite em questão, mas como ocorre na formulação de Cauchy, também não fornece meios para se determinar qual é o limite. Fazendo-se uso da proposição apresentada nesse artigo, a partir do momento que se determina que a oscilação extrema é vazia ou contém apenas um ponto, 
isto é, que o limite existe, pode-se determiná-lo achando o "supremo" da seção inferior extrema ou o "ínfimo" da seção superior extrema. A dificuldade do método de Russell consiste em determinar quais conjuntos são as seções inferiores e superiores, para posteriormente definir quais são as seções superiores e inferiores extremas. Por essas razões, foram explorados exemplos de funções nos quais isso se torna fácil, mas dependendo da função dada, isso também pode ser bem complicado.

Além das considerações quanto à dificuldade na aplicação de um método ou outro, cabe aqui observar também que o correto entendimento da ideia de Russell permite formar um juízo intuitivo sobre o limite, bem parecido com aquele que o "Teorema do Confronto"16 fornece, pois à medida que se determinam as seções inferiores, por exemplo, estabelece-se um limite superior para os valores que a função atinge em algum intervalo e, da mesma forma, as seções superiores fornecem um limite inferior para a função em algum intervalo. Dessa forma, se tanto esses limites superiores, quanto os limites inferiores se aproximam de um ponto quando os intervalos vão ficando menores, fazendo com que a oscilação extrema seja vazia ou contendo apenas um número, o limite existe.

Conclui-se, assim, com a reflexão de D’Ambrósio sobre o rigor na Matemática no Prefácio de seu livro Cálculo e Introdução à Análise, de 1975. O professor e historiador da Matemática ressalta que não se pode perder de vista ou muito menos abafar a intuição daqueles que estudam a Matemática em nome do rigor. Esse pensamento vai de encontro, filosoficamente falando, aos objetivos desse artigo que tem a preocupação em mostrar um novo ponto de vista para um velho assunto e, à própria ideia do limite russeliano quando esse é entendido corretamente. Nas palavras de D’Ambrósio:

"Apresentamos aqui um livro de cálculo dirigido ao aluno que espera seguir uma carreira em que a Matemática será amplamente utilizada ou mesmo ao que pretende seguir um curso de Matemática. O tratamento é rigoroso até o ponto em que preocupações de rigor não interfiram com as bases intuitivas do cálculo. Entendemos que sensibilidade para rigor matemático é algo que se adquire, que se sente após alguma vivencia com a Matemática, e que surge naturalmente com o desenvolvimento de uma certa 'intuição para o rigor'. Deste modo, tratar os diversos assuntos que aparecem no início de um curso de cálculo com o 'devido rigor' pode neutralizar o que nos parece a função essencial de um primeiro curso em qualquer assunto: despertar no leitor uma certa curiosidade e espírito inquisitivo que, aliado a algum gosto pelo assunto, o estimulará a procurar um tratamento mais rigoroso e profundo. Naturalmente, este tratamento, que de nenhum modo pode ser considerado 'não rigoroso', é em vários pontos dirigido a estimular o leitor a questionar sobre sua validade, sobre o seu grau de generalidade e sobre o potencial para aplicações e generalizações. Cumpre ao professor explorar ao máximo estas possibilidades e entrar em detalhes sobre os diversos pontos que

\footnotetext{
${ }^{16}$ Sejam $\left(a_{n}\right)$, $\left(b_{n}\right)$ e $\left(c_{n}\right)$, sequências tais que $\left(a_{n}\right) \leq\left(b_{n}\right)$ e $\left(c_{n}\right), \forall n \in \mathbb{N}$, se as sequências $\left(a_{n}\right)$ e $\left(c_{n}\right)$ tiverem um limite $L$ quando $n \rightarrow \infty$, então $(b n)$ também terá o limite $n \rightarrow+\infty$. (GUIDORIZZI, 2001 p.90)
}

RBHM, Vol. 16, n 31, p. 99-112, 2016 
tenham suscitado dúvida ou interesse dos alunos." (D'AMBROSIO, 1975, p. ix.)

\section{Bibliografia}

BARONI, Rosa Lúcia Sverzut \& OTERO-GARCIA, Sílvio César. 2014. Aspectos da História da Análise Matemática de Cauchy a Lebesgue. São Paulo: Cultura Acadêmica.

CAUCHY, Augustin-Louis. 1821. Cours d'Analyse de l'École Royale Polytechnique. Premier partie. Analyse algébrique. Paris: Chez Debure Frères.

D’AMBROSIO, Ubiratan. 1975. Cálculo e Introdução à Análise. São Paulo: Companhia Editora Nacional.

GRABINER, Judith. 2005. The origins of Cauchy's rigorous calculus. Mineola: Dover Publications.

GRATTAN-GUINNESS, Ivor. 2009. Routes of learning: highways, pathways, and byways in the history of mathematics. Baltimore: The Johns Hopkins University Press.

GUIDORIZZI, Hamilton. 2001 (5 ${ }^{\mathrm{a}}$. Ed.). Um curso de cálculo. Rio de Janeiro: LTC, 2002. KATZ, Victor. 2010. História da Matemática. Lisboa: Fundação Calouste-Gulbekian, 2010.

RUSSELL, Bertrand. 1966 (2ª Ed.). Introdução à Filosofia da Matemática. Rio de Janeiro: Zahar.

RUSSELL, Bertrand \& WHITEHEAD, Alfred North. 2013 (v1). Principia Mathematica. Volume One. San Bernardino: Rough Draft Printing. Principia Mathematica. 2013 (v2). San Bernardino: Rough Draft Printing.

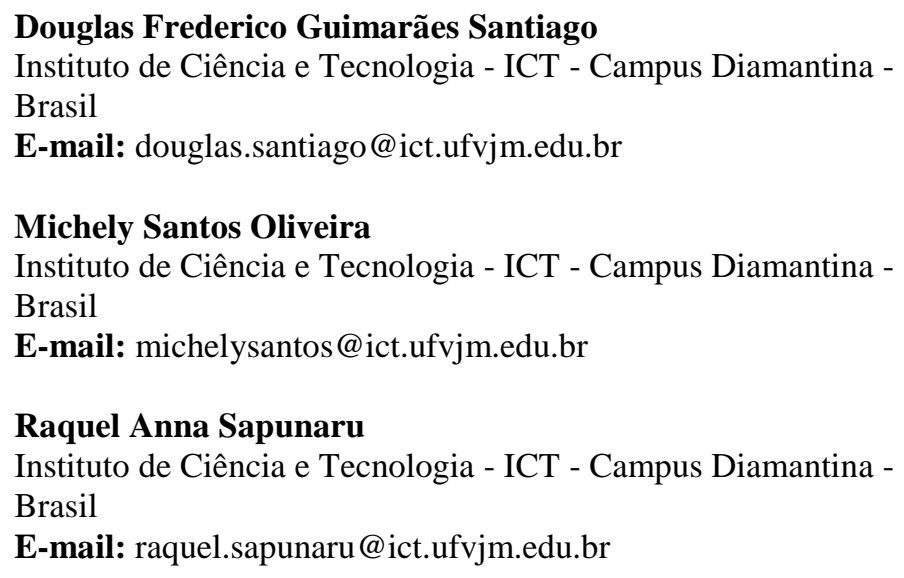

